\title{
Diagnosis Algorithms for a Symbolically Modeled Manufacturing Process
}

\author{
N. Rakoto-Ravalontsalama \\ Department of Automatic Control \\ Ecole des Mines de Nantes \\ 4, rue Alfred Kastler, F-44307 Nantes Cedex 03, France \\ rakotodauto.emn.fr
}

\begin{abstract}
The considered manufacturing process is modeled in both numerical and symbolic ways, in a disjoint manner. This paper focuses on the symbolic model of the given Discrete Event System (DES). A new method for diagnosing some symbolic parameters is presented. The proposed diagnosis algorithms are to be performed sequentially. The simulation results show that these algorithms have improved the symbolic part of the process model.
\end{abstract}

\section{Introduction}

Fault detection and isolation has received considerable attention in the literature because it is an important task in automatic control and complex systems. Many approaches have been applied to industrial large processes [11] or [2].

It is far from obvious to build up a complete model for a given process. Thus dealing with incomplete knowledge is quite relevant to knowledge representation. Various symbolic (qualitative) approaches have been proposed to cope with such an incomplete knowledge including sign algebra which is the most elementary (thus incomplete) way of representation.

Ambiguity is the predominant source of weakness in sign algebra and it has been a major focus of research in qualitative reasoning like FOG [12], O(M) [9], SR1 [5] and ROM(K) [3]. These are some examples of approaches that bring improvements with regard to the classical sign algebra.

This paper focuses on a particular Discrete Event System (DES) which is a manufacturing line. It transforms a raw material into a finished product. The system has a hierarchical structure that are subprocess, workcell and workstation. The process is composed of a set of sequential subprocesses. Each subprocess is made of a number of sequential workcells. Lastly each workcell is a set of sequential workstations. The workstation is the elementary component, typically a machine. It is modeled in both numerical and symbolic way, but in a disjoint manner (no model overlapping).

The aim of the paper is to enhance the symbolic model, especially by detecting and isolating the uncertain (or ambiguous) symbols. For the rest of the paper, uncertain and ambiguous have the same meaning that is the symbolic value $q$ ?

V.N. Alexandrov et al. (Eds.): ICCS 2001, LNCS 2073, pp. 1228-1236, 2001.

(c) Springer-Verlag Berlin Heidelberg 2001 
Then a diagnosis algorithm to deal with such an uncertain information is proposed. This is based on two sequential algorithms that are coarse diagnosis followed by fine diagnosis.

This paper is organized as follows: Section 2 briefly presents a summary of the previous process model, section 3 proposes the basic principles of the two diagnosis algorithms. A discussion is given in section 4 .

\section{Preliminaries}

The DES is a TV-tube manufacturing system. This has been modeled in order to build a hierarchical control of the whole process. The system has been decomposed using a hierarchical approach that are subprocess, workcell and workstation. Each component is made of sequential sets of sub-components. A basic specification has been defined for the most elementary element, i.e., the workstation. The basic model is summarized here: for more details see [7], [8], and [14].

A workstation is the most elementary machine, typically consisting of one processing tool, position or action. The task performed at this position is generally done by a robot. Firstly, the workstation model takes into account some properties of classical control systems like controllability and observability. Thus distinction has been made between control and uncontrollable parameters (generally inputs) as well as observable and unobservable parameters (mostly outputs). Secondly, the model is focused on the product itself, the TV-tube, but also includes some process parameters. Finally, each parameter can be of numerical or symbolic type. These types of specification are given as follows:

- Numerical (quantitative) behavioral model: it mostly consists of algebraic relations and ordinary differential equations. This type of model is recognized to be the best one when available. Some examples of significant numerical variables are the tube temperature or its rotation speed at a given machine.

- Symbolic (qualitative) behavioral model: it is used when the quantitative specification is not available, either when there is not enough information (most of the cases) or when the numerical information extraction is costly too expensive.

It can be noticed that the symbolic and numerical models are disjoint sets, but the two type of parameters interact. The symbolic specification is mainly used to deal with rough knowledge, which are represented by the following set of symbols $S=\left\{q_{-}, q_{0}, q_{+}\right\}$. The meaning of each symbol is as follows: the sign represents the deviation from the nominal value of a given variable. It is then obvious that the best process model corresponds to the one where all symbolic variables take their nominal value i.e., the symbolic value $q_{0}$. The sign addition and sign multiplication are given in Table 1. 
Table 1. Sign addition (left) and sign multiplication (right)

\begin{tabular}{c||c|c|c|c}
\hline$\oplus$ & $q_{-}$ & $q_{0}$ & $q_{+}$ & $q_{?}$ \\
\hline \hline$q_{-}$ & $q_{-}$ & $q_{-}$ & $q_{?}$ & $q_{?}$ \\
\hline$q_{0}$ & $q_{-}$ & $q_{0}$ & $q_{+}$ & $q_{?}$ \\
\hline$q_{+}$ & $q_{?}$ & $q_{+}$ & $q_{+}$ & $q_{?}$ \\
\hline$q_{?}$ & $q_{?}$ & $q_{?}$ & $q_{?}$ & $q_{?}$ \\
\hline
\end{tabular}

\begin{tabular}{c||c|c|c|c}
\hline$\otimes$ & $q_{-}$ & $q_{0}$ & $q_{+}$ & $q_{?}$ \\
\hline \hline$q_{-}$ & $q_{+}$ & $q_{0}$ & $q_{-}$ & $q_{?}$ \\
\hline$q_{0}$ & $q_{0}$ & $q_{0}$ & $q_{0}$ & $q_{0}$ \\
\hline$q_{+}$ & $q_{-}$ & $q_{0}$ & $q_{+}$ & $q_{?}$ \\
\hline$q_{?}$ & $q_{?}$ & $q_{0}$ & $q_{?}$ & $q_{?}$ \\
\hline
\end{tabular}

This symbolic model seemed to be not enough to model a part of the DES. Thus the classical set $S$ has been extended to a set $S^{\prime \prime}$ by adding an ambiguous value $\left(q_{\text {? }}\right)$ and all alternate values $S_{d}=\left\{\left(q_{-}, q_{0}\right),\left(q_{0}, q_{+}\right),\left(q_{-}, q_{+}\right)\right\}$. These represent the binary logical OR of two basic symbols. For details on the symbolic specification, see [13] and [14].

- Sign algebra set $S=\left\{q_{-}, q_{0}, q_{+}\right\}, S^{\prime}=S \cup\left\{q_{\text {? }}\right\}$

- Alternate value $\left(q_{a}, q_{b}\right)=q_{a} \vee q_{b}$ (logical-OR of $q_{a}$ and $\left.q_{b}\right)$.

$-S_{d}=\left\{\left(q_{-}, q_{0}\right),\left(q_{0}, q_{+}\right),\left(q_{-}, q_{+}\right)\right\}$

- Universe of calculus: $S^{\prime \prime}=S^{\prime} \cup S_{d}$

Using the set $S^{\prime \prime}$, some basic mappings like Qplusp(), Plusp() and Distp() have been defined. The addition operation extended to set $S^{\prime \prime}$ is shown in Table 2 and the multiplication operator extended to set $S^{\prime \prime}$ is given in Table 3 .

Table 2. Sign addition extended to set $S^{\prime \prime}$

\begin{tabular}{c||c|c|c|c|c|c|c}
\hline$\oplus$ & $q_{-}$ & $\left(q_{-}, q_{0}\right)$ & $q_{0}$ & $\left(q_{0}, q_{+}\right)$ & $q_{+}$ & $\left(q_{-}, q_{+}\right)$ & $q_{?}$ \\
\hline \hline$q_{-}$ & $q_{-}$ & $q_{-}$ & $q_{-}$ & $q_{?}$ & $q_{?}$ & $q_{?}$ & $q_{?}$ \\
\hline$\left(q_{-}, q_{0}\right)$ & $q_{-}$ & $\left(q_{-}, q_{0}\right)$ & $\left(q_{-}, q_{0}\right)$ & $q_{?}$ & $q_{?}$ & $q_{?}$ & $q_{?}$ \\
\hline$q_{0}$ & $q_{-}\left(q_{-}, q_{0}\right)$ & $q_{0}$ & $\left(q_{0}, q_{+}\right)$ & $q_{+}$ & $\left(q_{-}, q_{+}\right)$ & $q_{?}$ \\
\hline$\left(q_{0}, q_{+}\right)$ & $q_{?}$ & $q_{?}$ & $\left(q_{0}, q_{+}\right)$ & $\left(q_{0}, q_{+}\right)$ & $q_{+}$ & $q_{?}$ & $q_{?}$ \\
\hline$q_{+}$ & $q_{?}$ & $q_{?}$ & $q_{+}$ & $q_{+}$ & $q_{+}$ & $q_{?}$ & $q_{?}$ \\
\hline$\left(q_{-}, q_{+}\right)$ & $q_{?}$ & $q_{?}$ & $\left(q_{-}, q_{+}\right)$ & $q_{?}$ & $q_{?}$ & $q_{?}$ & $q_{?}$ \\
\hline$q_{?}$ & $q_{?}$ & $q_{?}$ & $q_{?}$ & $q_{?}$ & $q_{?}$ & $q_{?}$ & $q_{?}$ \\
\hline
\end{tabular}

Finally, by combining some basic mappings which are associated with the sign operators $\oplus$ and $\ominus$, some compound mappings like (Plus(), Dist(), Tune()) have been defined. The basic and compound mappings definitions are specified in Table 4. For example, the compound mapping Plus () is a combination of two basic mappings plusp() obtained with a subtraction operator. The meaning of each mapping and more details on the symbolic specification can be found in [13] and [14]. 
Table 3. Sign multiplication extended to set $S^{\prime \prime}$

\begin{tabular}{c||c|c|c|c|c|c|c}
\hline$\otimes$ & $q_{-}$ & $\left(q_{-}, q_{0}\right)$ & $q_{0}$ & $\left(q_{0}, q_{+}\right)$ & $q_{+}$ & $\left(q_{-}, q_{+}\right)$ & $q_{?}$ \\
\hline \hline$q_{-}$ & $q_{+}$ & $q_{0}, q_{+}$ & $q_{0}$ & $q_{-}, q_{0}$ & $q_{-}$ & $q_{-}, q_{+}$ & $q_{?}$ \\
\hline$\left(q_{-}, q_{0}\right)$ & $q_{0}, q_{+}$ & $\left(q_{0}, q_{+}\right)$ & $q_{0}$ & $\left(q_{-}, q_{0}\right)$ & $\left(q_{-}, q_{0}\right)$ & $q_{?}$ & $q_{?}$ \\
\hline$q_{0}$ & $q_{0}$ & $q_{0}$ & $q_{0}$ & $q_{0}$ & $q_{0}$ & $q_{0}$ & $q_{0}$ \\
\hline$\left(q_{0}, q_{+}\right)$ & $q_{-}, q_{0}$ & $q_{-}, q_{0}$ & $q_{0}$ & $\left(q_{0}, q_{+}\right)$ & $\left(q_{0}, q_{+}\right)$ & $q_{?}$ & $q_{?}$ \\
\hline$q_{+}$ & $q_{-}$ & $\left(q_{-}, q_{0}\right)$ & $q_{0}$ & $\left(q_{0}, q_{+}\right)$ & $q_{+}$ & $\left(q_{-}, q_{+}\right)$ & $q_{?}$ \\
\hline$\left(q_{-}, q_{+}\right)$ & $\left(q_{-}, q_{+}\right.$ & $q_{?}$ & $q_{0}$ & $q_{?}$ & $\left(q_{-}, q_{+}\right)$ & $\left(q_{-}, q_{+}\right)$ & $q_{?}$ \\
\hline$q_{?}$ & $q_{?}$ & $q_{?}$ & $q_{0}$ & $q_{?}$ & $q_{?}$ & $q_{?}$ & $q_{?}$ \\
\hline
\end{tabular}

Table 4. Basic and compound symbolic mappings

\begin{tabular}{|c|c|c|c|c|}
\hline Mappings & Initial set(s) & Final : & Expression & \\
\hline Qlusp $(s)$ & $S^{\prime \prime}$ & $S^{\prime \prime}$ & $=\left\{\begin{array}{l}q_{0} \\
q_{+} \\
\left(q_{0}, q_{+}\right)\end{array}\right.$ & $\begin{array}{l}\text { if }\left(s=q_{-} \text {or } s=\left(q_{-}, q_{0}\right) \text { or } s=q_{0}\right) \text {, } \\
\text { if } s=q_{+}, \\
\text {otherwise. }\end{array}$ \\
\hline $\operatorname{Plusp}(x, \Delta)$ & $R \times R$ & $S^{\prime \prime}$ & $=\left\{\begin{array}{l}q_{0} \\
\left(q_{0}, q_{+}\right) \\
q_{+}\end{array}\right.$ & $\begin{array}{l}\text { if } x \leq 0 \\
\text { if } 0<x \leq \Delta \\
\text { if } x>\Delta\end{array}$ \\
\hline $\operatorname{Distp}(x, \Delta)$ & $R \times R$ & $S^{\prime \prime}$ & $=\left\{\begin{array}{l}q_{0} \\
q_{?} \\
\left(q_{-}, q_{+}\right)\end{array}\right.$ & $\begin{array}{l}\text { if } x \leq 0 \\
\text { if } 0<x \leq \Delta \\
\text { if } x>\Delta\end{array}$ \\
\hline $\operatorname{Plus}\left(x, \Delta_{1}, \Delta_{2}\right)$ & $R \times R \times R$ & $S^{\prime \prime}$ & \multicolumn{2}{|c|}{$=\operatorname{Plusp}\left(x-\Delta_{1}, \Delta_{2}\right) \ominus \operatorname{Plusp}\left(-x-\Delta_{1}, \Delta_{2}\right)$} \\
\hline $\operatorname{Dist}\left(x, \Delta_{1}, \Delta_{2}\right)$ & $R \times R \times R$ & $S^{\prime \prime}$ & \multicolumn{2}{|c|}{$=\operatorname{Distp}\left(x-\Delta_{1}, \Delta_{2}\right) \oplus \operatorname{Distp}\left(-x-\Delta_{1}, \Delta_{2}\right)$} \\
\hline Tune $\left(x, \Delta_{1}, \Delta_{2}\right)$ & $R \times R \times R$ & $S^{\prime \prime}$ & \multicolumn{2}{|c|}{$=\operatorname{Plusp}\left(x-\Delta_{1}, \Delta_{2}\right) \oplus \operatorname{Plusp}\left(-x-\Delta_{1}, \Delta_{2}\right)$} \\
\hline
\end{tabular}




\section{Diagnosis}

Fault diagnosis generally consists in fault detection and then isolation. In this work, fault is not the "usual" failure that can be found in industrial complex systems but the ambiguous symbolic variable $q$ ?

We recall that the symbolic knowledge is expressed by the sign algebra set, $S_{d}=\left\{q_{-}, q_{0}, q_{+}\right\}$, to which additional symbols have been added. These are alternate symbols, $S_{d}=\left\{\left(q_{-}, q_{0}\right),\left(q_{0}, q_{+}\right),\left(q_{-}, q_{+}\right)\right\}$. Furthermore, once an ambiguous symbol $q$ ? appears, it is propagated to another related variables, due to the sequential structure of the DES. The objective of the diagnostic task is to enhance the symbolic model that is to remove all the ambiguous and all the alternate symbols. Thus the target set after diagnosis is the set of sign algebra, $S_{f}=S=\left\{q_{-}, q_{0}, q_{+}\right\}$. The ideal case would be that this target set is reduced to the single null element $\left\{q_{0}\right\}$.

Therefore, to improve the model i.e., to remove all ambiguous and alternate values, two simple sequential algorithms, coarse diagnosis and fine diagnosis are proposed. The first algorithm is devoted to the treatment of variables taking the ambiguous value $q$ ? while the second one deals with the alternate symbols (belonging to Set $S_{d}$ ). These algorithms are to be performed sequentially, coarse diagnosis first, followed by the fine one.

\subsection{Preliminary Assumptions}

Some assumptions have to be made before performing such algorithms:

1. There exists at least one symbolic variable taking the ambiguous value $q$ ? at the beginning of the simulation. Every symbolic variable taking such a value is called an uncertain variable.

2. According to the structure of the DES and because of the use of the addition operation, an uncertain variable will always forward its uncertain value to every related variable in the next workstations ${ }^{1}$.

3. For each uncertain variable, there exists at least one control action in order to change its value; this (these) control variable(s) is (are) located only in the same workstation - not general control variable(s).

4. Each control variable is numerical and is bounded, $u \in\left[u_{\min }, u_{\max }\right]$.

5. Coarse diagnosis as well as fine diagnosis are performed off-line.

\subsection{Preliminary Causal Graph}

The building of the hybrid causal graph (HCG) is also required, before performing the diagnostic task. One HCG is built for each workstation. The causal graph is a set of points (nodes) and a set of arcs (relations) connecting some nodes. A node is a real-valued or symbolic variable $\left(x_{i} \in R \cup S^{\prime \prime}\right)$. A directed arc represents a relation between the connected variables, from the cause(s) to the effect(s). A numerical are is an arc where both cause(s) and effect(s) are numerical. A

\footnotetext{
${ }^{1}$ This is not true if the Q-multiplication is used, because $q_{\text {? }} \otimes q_{0}=q_{0}$.
} 
symbolic arc is an arc for which the effect variable(s) is (are) symbolic, the cause(s) being numerical or symbolic.

Existing similar approaches are SDG, Signed Directed Graph introduced by Iri et al. [6], ESDG, Enhanced Signed Directed Graphs proposed by Oyeleye and Kramer [10] and QUAF, Qualitative Analysis of Feedback, proposed by Rose and Kramer [15].

\subsection{Coarse Diagnosis}

The coarse diagnosis objective is to remove the uncertain variables from the initial set $S^{\prime \prime}$. The algorithm consists in the isolation of the uncertain variable, then the tuning of the control variable and performing the simulation. Lastly other uncertain(s) variable(s) are then detected. The target set is the initial set without the ambiguous values.

- Initial set: $S_{i}=S^{\prime \prime}$

- Objective: to remove all uncertain variables (ambiguous values $q$ ?)

- Target set: $S_{f}=S^{\prime \prime}-\left\{q_{\text {? }}\right\}$; Ideal case $S_{f}=\left\{q_{0}\right\}$.

- Algorithm: isolation, tuning control variable, simulation and detection

More precisely the detailed algorithm to perform such a diagnosis task is given below:

Coarse Diagnosis

1. Initialization:

a. Isolate the first subprocess

b. Isolate the first workcell

c. Isolate the first workstation

d. Isolate the first uncertain variable i.e. $q$ ?

2. Isolation of uncertain variable:

a. Isolate the next subprocess

b. Isolate the next workcell

c. Isolate the next workstation

d. Isolate the next uncertain variable i.e. q?

3. Performing control action:

a. find appropriate control variable (control tuning)

b. determine its numerical value

c. apply control action

4. Running simulation:

5. Detection of (other) uncertain variable(s)

a. if analyzed qvar is still uncertain then goto $3 \mathrm{~b}$.

---- else goto $5 b$.

b. if at least one uncertain variable is detected in current workstation then goto $1 \mathrm{~d}$.

---- else goto $5 \mathrm{c}$.

c. if at least one uncertain variable is detected in current workcell then goto $2 c$.

--- else goto $5 d$. 
d. if at least one uncertain variable is detected in current subprocess then goto $2 b$.

--- else goto $5 \mathrm{e}$.

e. if at least one uncertain variable is detected in the process then goto

2 a. else end.

Some remarks can be made upon this coarse diagnosis algorithm:

- The considered uncertain variable is assumed to be an output variable (and not a process one); uncertain process variable always gives rise to uncertain output variable(s) in the same workstation ${ }^{2}$.

- It is assumed that there exists at least one uncertain variable at the beginning of the diagnosis task so that the algorithm begins with isolation instead with detection (unlike standard diagnosis: detection, then isolation)

\subsection{Fine Diagnosis}

- Initial set: $S_{i}=S^{\prime \prime}-\left\{q_{?}\right\}$

- Objective: to remove all alternate variables $\left(S_{d}\right)$

- Target set: $S_{f}=S_{i}-S_{d}=S$; Ideal case: $S_{f}=\left\{q_{0}\right\}$

- Algorithm: isolation, tuning control variable, simulation and detection

The fine diagnosis algorithm is exactly the same algorithm as for coarse diagnosis, but the focus is on alternate variables $\left(S_{d}\right)$ instead of ambiguous ones $\left\{q_{\text {? }}\right\}$.

\section{Discussion}

The numerical model is represented by ordinary differential equations, while the symbolic model is expressed by algebraic sign relations and decision tables with if-then rules in Table 1, Table 2 and Table 3 . The simulation duration is about 1 second while the effective time is 20 minutes. The simulator writes the output variables in a specific file.

The diagnosis algorithms have been applied to a part of the manufacturing system. This system has strong sequential properties. The simulation has been performed to the Flowcoat Green workcell, which is a sequential collection of twelve workstations, FG01 to FG12. The main objective of this workcell is to put a green flowcoat liquid on a blank TV tube. A significant workstation is the fourth one, FG04. This workstation is recognized to be significant because at this location is determined the amount of green flowcoat liquid to be spread which will become the green phosphor lines on the finished TV tube.

The coarse diagnosis has detected 10 ambiguous variables, while the fine algorithm have removed 7 alternate symbols. The two diagnosis algorithms have improved the model in the way that the refined model has a reduced number of uncertain variables, thus reducing the total number of states of the hybrid process. However, the proposed method only deal with the symbolic model, that is only a part of the complete model. And the number of initial ambiguous

\footnotetext{
${ }^{2}$ This holds since no Q-multiplication is used in the present formalism.
} 
variables is relatively low because the diagnosis algorithms have been applied to a model that has been previously tuned (with other methods).

However, many assumptions have been made. The propagation of the ambiguous symbolic variable $q$ ? is observable since only the sign addition operator is used for the symbolic model. Indeed, if the symbolic model include the use of the multiplication operator, an ambiguous symbolic variable $q$ ? could be transformed to $q_{0}$, thus making this failure unobservable. Another restrictive assumption is the existence of a numerical control action that can be tuned in order to remove the ambiguous variable. This could be quite difficult to obtain.

\section{Concluding Remarks}

A new method of diagnosing uncertain parameters has been presented. The system is modeled in a disjoint numerical and symbolic way. The main idea is to detect uncertain (or ambiguous) variables in order to improve the symbolic discrete event model. The advantage of such method is the systematic checking of uncertain variables thanks to the symbolic model of the system and also to the sequential structure of the class of the considered discrete event system.

However the extension of this approach to another class of discrete event systems is not straightforward because many assumptions have been made. For example the existence of a control action that can tune the model in order to remove the ambiguous symbolic variable is not always guaranteed. The type of considered DES is quite a simple one. Fault diagnosis in FlowShop or JobShop systems become more complicated due to the multiplicity of machines thus a multiplicity of possible faults.

On-going work concerns the automatic tuning of control variable using lower and upper bounds, where some process expertise is required. Finally the next step is the automated diagnosis of such algorithms in order to perform the diagnostic task on-line.

\section{References}

1. A. Aghasaryan, E. Fabre, A. Benveniste, R. Boubour and C. Jard "A Petri net approach to fault detection and diagnosis in distributed systems: Extending Viterbi algorithm and HMM techniques to Petri nets". In Proc. 36th IEEE Conf. on Decision and Control, Dec. 1997.

2. R. Boubour, C. Jard, A. Aghasaryan, E. Fabre and A. Benveniste. "A Petri net approach to fault detection and diagnosis in distributed systems: Application to telecommunication networks, motivations, and modelling". In Proc. 36th IEEE Conf. on Decision and Control, Dec. 1997.

3. P. Dague. "Symbolic reasoning with relative orders of magnitude". In Proc. 13th Int. Conf. on Artificial Intelligence IJCAI'93, pp. 1509-1514, Chambery, France, 1993. 
4. R. Debouk, S. Lafortune and D. Teneketzis. "Coordinated decentralized protocols for failure diagnosis of discrete event systems". In Proc. 37th IEEE Conf. on Decision and Control, Dec. 1998.

5. J. de Kleer and B.C. Williams. "Diagnosing multiple faults". Artificial Intelligence., 32:97-130, 1987.

6. M. Iri, K. Aoki, E. O'Schima and H. Matsuyama." An algorithm for diagnosis of system failure in the chemical process". Computer Chemical Engineering, 3:489-493, 1979 .

7. J. Kamerbeek and J.S. Kikkert. "Esprit 2428 IPCES: Process description of prototype 2". Private Communication, Philips-PRL and Philips-TCDC, Eindhoven, The Netherlands, December 1991.

8. J. Kamerbeek. "Generating simulators from causal process knowledge". Proc. of European Simulation Symposium 1993. Delft, The Netherlands, October 1993.

9. M.L. Mavrovouniotis and G. Stephanopoulos. "Formal order of magnitude reasoning in process engineering". Computer Chemical Engineering, 12:867880,1988 .

10. O.O. Oyeleye and M.A. Kramer. "Qualitative simulation of chemical process systems: Steady-state analysis". A IChE Journal, 34:1441-1454, 1988.

11. P.M. Frank. "Analytical and qualitative model-based fault diagnosis - A survey and some new results". European Journal of Control, 2:6-28, 1996.

12. O. Raiman. "Order of magnitude reasoning". Proc. of AAAI-86, pp. 100104, Philadelphia, PA, USA . 1986.

13. N. Rakoto-Ravalontsalama, A. Missier, and J.S. Kikkert. "Qualitative operators and process engineer semantics of uncertainty". In B. Bouchon-Meunier, L. Valverde, and R.R. Yager, editors, Lecture Notes in Computer Science 682, IPMU'92 - Advanced Methods in Artificial Intelligence, pp. 284-293. Springer Verlag, 1992.

14. N. Rakoto-Ravalontsalama and J. Aguilar-Martin. "Knowledge-based modelling of a TV-tube manufacturing system" IFAC Journal of Control Engineering Practice, 4(1), pp. 117-123, Jan. 1996.

15. P. Rose and M.A. Kramer. "Qualitative analysis of causal feedback". Proc. of AAAI-91, Anaheim, CA, USA 1991.

16. M. Sampath, S. Lafortune, and D. Teneketzis. "Active diagnosis of discrete event systems". IEEE Trans. Automat. Contr., 43(7), pp. 908-929, July 1998. 